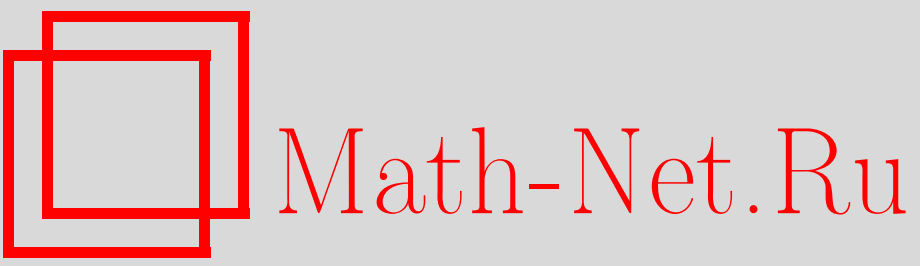

О. И. Завьялов, О нелинейных представлениях группы Лоренца в квантовой теории поля, ТМФ, 2001, том 127, номер 1, 75-89

DOI: https://doi.org/10.4213/tmf449

Использование Общероссийского математического портала Math-Net.Ru подразумевает, что вы прочитали и согласны с пользовательским соглашением

http://www . mathnet.ru/rus/agreement

Параметры загрузки:

IP : 3.85.7.115

26 апреля 2023 г., 18:05:34 
ТЕОРЕТИЧЕСКАЯ

И МАТЕМАТИЧЕСКАЯ

ФИЗИКА

Том 127, № 1

апрель, 2001

(C) 2001 г

О.И. Завьялов*

\section{О НЕЛИНЕЙНЫХ ПРЕДСТАВЛЕНИЯХ ГРУППЫ ЛОРЕНЦА В КВАНТОВОЙ ТЕОРИИ ПОЛЯ}

\footnotetext{
Формулы для нелинейного представления группы Лоренца обобщены на случай волновых функций вигнеровских частиц, траектории которых начинаются в точках фазового пространства с ненулевой координатой.
}

\section{1. ВВЕДЕНИЕ}

В нашей работе [1] показано, что требование, чтобы функция Вигнера, обобщенная на случай свободных частиц с "релятивистским" гамильтонианом $H(\mathbf{p})=\sqrt{\mathbf{p}^{2}+m^{2}}$, преобразовывалась при лоренцевых преобразованиях как истинная плотность распределения вигнеровских псевдочастищ в фазовом пространстве, приводит к нелинейному закону преобразования (если он сушествует) для "релятивистских" волновых функций. В двумерном пространстве-времени инфинитезимально (т.е. при бесконечно малых двумерных "бустах") упомянутая нелинейность связана с тем обстоятельством, что при двумерном "бустировании" особым образом меняется на массовом гиперболоиде импульсная переменная $\gamma$ для вигнеровских псевдочастиц, даже в том случае, когда соответствующая траектория исходит из точки с нулевой координатой $q=0$, каковое условие, естественно, сохраняется и в результате "бустирования". Именно такое специально редуцированное условие лоренц-инвариантности было использовано в [1]. В настояшей работе мы обобшаем формулу для нелинейных инфинитезимальных преобразований волновых функций на случай, когда траектория начинается в точке фазового пространства с ненулевой координатой $q$, т.е. когда $q$ также меняется при двумерных "бустах". Таким образом, утверждается, во-первых, что такие преобразования действительно существуют, а во-вторых, что новый закон преобразования учитывает теперь полную информацию о лоренц-инвариантности соответствующей функции Вигнера. Обший вид таких нелинейных инфинитезимальных преобразований (хотя и в несколько неявной форме) будет описан в нашей другой заметке. Чтобы испробовать примененную технику на простом примере, мы сначала рассмотрим вопрос о правилах преобразования нерелятивистской волновой функции по отношению к группе Галилея.

* Математический институт им. В. А. Стеклова РАН, Москва, Россия 
Кроме того, мы приведем здесь новое координатное представление для нерелятивистской функции Вигнера и на этой основе передокажем главные свойства галилеевой версии $W_{\text {нер }}$ функции Вигнера. Будут построены глобальные преобразования волновых функций относительно этой группы. Мы обсудим также соответствующую программу действий применительно к группе Лоренца.

В статье [2] была построена функция $W(\mathbf{p}, \mathbf{q} ; t)$, которая, по-видимому, играет в "релятивистской” квантовой механике свободных частиц, т.е. частиц с гамильтонианом

$$
H(\mathbf{p})=\omega(\mathbf{p})=\sqrt{\mathbf{p}^{2}+m^{2}},
$$

ту же роль, что и известная функция Вигнера (см. [3] и [4]) для нерелятивистских свободных частиц, в которую она, собственно, и переходит при малых скоростях. Имеем

$$
\begin{aligned}
W(\mathbf{p}, \mathbf{q} ; t)= & \frac{1}{(2 \pi)^{3}} \int d \mathbf{p}_{1} d \mathbf{p}_{2} \psi^{*}\left(\mathbf{p}_{1}\right) \psi\left(\mathbf{p}_{2}\right) \delta\left(\mathbf{p}-\left(\mathbf{p}_{1} \dot{+} \mathbf{p}_{2}\right)\right) \times \\
& \times \exp \left(i\left(\omega\left(\mathbf{p}_{1}\right)-\omega\left(\mathbf{p}_{2}\right)\right) t+i\left(\mathbf{p}_{2}-\mathbf{p}_{1}\right) \mathbf{q}\right)
\end{aligned}
$$

где символ “+” обозначает специальную сумму на массовом гиперболоиде:

$$
\mathbf{p}_{1}+\mathbf{p}_{2}=m \frac{\mathbf{p}_{1}+\mathbf{p}_{2}}{\sqrt{2\left(m^{2}+\omega\left(\mathbf{p}_{1}\right) \omega\left(\mathbf{p}_{2}\right)-\mathbf{p}_{1} \mathbf{p}_{2}\right)}},
$$

$\psi(\mathbf{p})$ - волновая функция частицы в импульсном представлении в нулевой момент времени.

Особо следует отметить, что координаты q входят в функцию Вигнера $W(\mathbf{p}, \mathbf{q} ; 0)$, заданную формулой (1), только через стандартный фазовый множитель

$$
E=\exp \left(i\left(\mathbf{p}_{2}-\mathbf{p}_{1}\right) \mathbf{q}\right)
$$

(мы будем называть его экспоненциальным координатным множителем), т.е., казалось бы, совершенно независимо от квантовой характеристики частицы - ее волновой функции. Мы увидим, однако, что квантовое описание и эта, так сказать, классическая трактовка функции Вигнера оказываются удивительным образом согласованньми.

В пределе малых импульсов $\mathbf{p}_{1,2}$ мы приходим к знакомому нерелятивистскому выражению для функции Вигнера:

$$
\begin{aligned}
W_{\text {нер }}(\mathbf{p}, \mathbf{q} ; t)= & \frac{1}{(2 \pi)^{3}} \int d \mathbf{p}_{1} d \mathbf{p}_{2} \psi^{*}\left(\mathbf{p}_{1}\right) \psi\left(\mathbf{p}_{2}\right) \delta\left(\mathbf{p}-\frac{\mathbf{p}_{1}+\mathbf{p}_{2}}{2}\right) \times \\
& \times \exp \left\{i\left(\frac{\mathbf{p}_{1}^{2}}{2 m}-\frac{\mathbf{p}_{2}^{2}}{2 m}\right) t+i\left(\mathbf{p}_{2}-\mathbf{p}_{1}\right) \mathbf{q}\right\}
\end{aligned}
$$

Эта величина может быть интерпретирована (см. обзор [5]) как “псевдоплотность" вероятностного распределения в шестимерном фазовом пространстве $\{\mathbf{p}, \mathbf{q}\}$ для ансамбля свободных классических нерелятивистских частиц, соответствующих гамильтониану

$$
H=\frac{\mathbf{p}^{2}}{2 m} \text {. }
$$


Легко проверить, что средние значения $\overline{f(\mathbf{q})}$ и $\overline{\varphi(\mathbf{p})}$ функций $f(\mathbf{q})$ и $\varphi(\mathbf{p})$ по этому классическому ансамблю совпадают с соответствующими квантовыми матричными элементами:

$$
\begin{aligned}
& \overline{f(\mathbf{q})}=\int d \mathbf{p} d \mathbf{q} W_{\mathrm{нер}}(\mathbf{p}, \mathbf{q} ; t) f(\mathbf{q})=\int d \mathbf{q} \widetilde{\psi}^{*}(\mathbf{q}, t) f(\hat{\mathbf{q}}) \widetilde{\psi}(\mathbf{q}, t), \\
& \overline{\varphi(\mathbf{p})}=\int d \mathbf{p} d \mathbf{q} W_{\mathrm{нер}}(\mathbf{p}, \mathbf{q} ; t) \varphi(\mathbf{p})=\int d \mathbf{p} \psi^{*}(\mathbf{p}, t) \varphi(\hat{\mathbf{p}}) \psi(\mathbf{p}, t),
\end{aligned}
$$

где

$$
\widetilde{\psi}(\mathbf{q}, t)=\frac{1}{(2 \pi)^{3 / 2}} \int d \mathbf{p} e^{i \mathbf{p q}} \psi(\mathbf{p}, t)
$$

и

$$
\psi(\mathbf{p}, t)=e^{-i \frac{\mathbf{p}^{2}}{2 m} t} \psi(\mathbf{p})
$$

- волновые функции соответственно в координатном и импульсном представлениях в момент времени $t$. Более того, среднее значение функции $F(\mathbf{p}, \mathbf{q})$ по этому классическому ансамблю совпадает с матричным элементом $\langle\widehat{F}(\hat{\mathbf{p}}, \hat{\mathbf{q}})\rangle$, где $\widehat{F}$-вейлевское квантование классической функции $F$.

Привлекательность вигнеровского подхода состоит, таким образом, именно в том, что квантовая эволюция и эволюция классического ансамбля тождественны. Однако эту тождественность не следует понимать слишком буквально: функция Вигнера не обязательно положительна. Это, собственно, мы и имели в виду, употребляя слово "псевдоплотность" вместо слова "плотность".

Перечислим теперь главные свойства нерелятивистской функции Вигнера (3):

1) она билинейна по волновой функции $\psi(\mathbf{p})$;

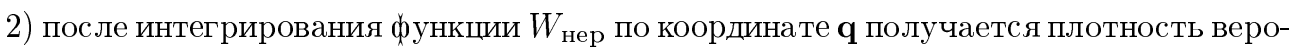
ятности в импульсном пространстве:

$$
\psi^{*}(\mathbf{p}, t) \psi(\mathbf{p}, t)=\int d \mathbf{q} W_{\text {нер }}(\mathbf{p}, \mathbf{q} ; t)
$$

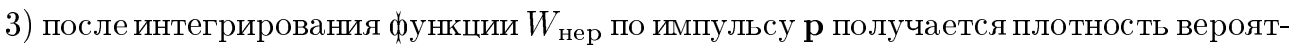
ности в координатном пространстве:

$$
\widetilde{\psi}^{*}(\mathbf{q}, t) \widetilde{\psi}(\mathbf{q}, t)=\int d \mathbf{p} W_{\text {нер }}(\mathbf{p}, \mathbf{q} ; t) ;
$$

4) функция Вигнера допускает классический закон эволюции, имитируюший закон эволюции вероятностной плотности распределения свободных частиц в фазовом пространстве:

$$
W_{\text {нер }}(\mathbf{p}, \mathbf{q} ; t+\tau)=W_{\text {нер }}\left(\mathbf{p}, \mathbf{q}-\frac{\mathbf{p}}{m} \tau ; t\right) .
$$

В работе [2] были доказаны аналоги этих равенств и для упомянутого релятивистского обобщения функции Вигнера. В следуюшем разделе мы передокажем эти свойства 
в нерелятивистском случае на новой основе так назьваемого координатного представления для функции $W_{\text {нер. }}$ Что касается уравнения $(8)$, то его релятивистский аналог (см. [2]) имеет форму

$$
W(\mathbf{p}, \mathbf{x} ; t+\tau)=W\left(\mathbf{p}, \mathbf{x}-\frac{\mathbf{p}}{\omega(\mathbf{p})} \tau ; t\right) .
$$

Разумеется, ситуация с физической интерпретацией релятивистской функции $W$ нисколько не лучше, чем в нерелятивистском случае. Эта функция также может быть отрицательной. Далее, средние значения по классическому ансамблю для "смешанных" функций (зависящих одновременно от координат и импульсов) не имеют ничего общего с квантовыми матричными элементами, по крайней мере в рамках вейлевского квантования. Таким образом, если считать, что квантовая механика - это именно вейлевское квантование, то приходится признать, что подход, базируюшийся на функции Вигнера (1), не имеет прямого отношения к релятивистской квантовой механике. С другой стороны, можно предполагать, что само усреднение по классическому ансамблю с (псевдо)вероятностью (1) определяет некий (вообще говоря, не обязательно вейлевский, но совпадаюший с вейлевским на функциях, зависяших только от $\hat{\mathbf{p}}$ или только от $\hat{\mathbf{q}}$ ) способ упорядочения операторов $\hat{\mathbf{p}}$ и $\hat{\mathbf{q}}$ в алгебре наблюдаемых, заданной этими образуюшими, так что рассматриваемая схема окажется просто одним из допустимых вариантов квантовой механики. Укажем здесь еше раз (см. [2]), что обшие принципы классической механики требуют таких трансформационных свойств функции $W(\mathbf{p}, \mathbf{x} ; t)$ по отношению к преобразованиям Лоренца, которые никоим образом не вытекают из общепринятого закона преобразования волновых функций. Это показывает также, что даже для случая свободных частиц несовместимость принципов квантовой механики со специальной теорией относительности, возможно, является более глубокой, чем обычно принято думать.

В настоящей работе мы попытаемся, однако, встать на противоположную точку зрения: мы попробуем найти такие преобразования волновых функций, которые приводят к "несофистицированным” “ожидаемым” трансформационным свойствам функции Вигнера (1). Мы, конечно, увидим опять, что такие преобразования с необходимостью являются нелинейными (они все-таки сушествуют), по крайней мере в двумерном пространстве-времени. Независимо от того, можно ли комбинацию функций (1) считать релятивистской функцией Вигнера, эта точка зрения кажется нам интересной по следующим причинам. Комбинация волновых функций (1) задает семейство траекторий воображаемых вигнеровских частиц, причем это совершенно не связано с наивной “псевдовероятностной” интерпретацией величины $W(\mathbf{p}, \mathbf{q} ; t)$. Каждая из этих траекторий порождает непрерывную последовательность каких-то (сейчас несушественно, каких именно) реальных событий в четырехмерном пространстве-времени. Четырехмерные координаты этих событий в любом случае должны меняться при изменении системы отсчета по стандартным законам Эйнштейна вне зависимости от того, имеет ли какой-нибудь смысл “простая" вигнеровская интерпретация (если только в теории свободных частиц вообще имеет смысл понятие "релятивистская волновая функция" и комбинацию $W(\mathbf{p}, \mathbf{q} ; t)$ в принципе можно измерить экспериментально). Если и впрямь 
в упомянутых нелинейных преобразованиях есть какой-то физический смысл и они конкурентоспособны по отношению к обычным линейным преобразованиям, то они могут сыграть неожиданную и, вернее всего, разрушительную роль для стандартной квантовой теории поля. В частности, операторы $a^{+}(\mathbf{p})$ и $a(\mathbf{p})$ рождения и уничтожения частиц с импульсом $\mathbf{p}$, входящие в выражение для обычного поля

$$
\varphi(x)=\frac{1}{\sqrt{2(2 \pi)^{3}}} \int \frac{d \mathbf{k}}{\omega(\mathbf{k})}\left[a^{+}(\mathbf{k}) e^{i \omega(\mathbf{k}) x^{0}-i \mathbf{k} \mathbf{x}}+a(\mathbf{k}) e^{-i \omega(\mathbf{k}) x^{0}+i \mathbf{k} \mathbf{x}}\right]
$$

в рамках этой схемы изменят (непонятным пока образом) свои трансформационные свойства. Останется ли при этом $\varphi(x)$ скалярным полем? Если нет, то какой объект получит роль скалярного поля? Эти и другие “диссидентские" вопросы мы собираемся обсудить в последуюших публикациях. Ниже мы ограничимся рассмотрением только двумерной модели пространства-времени. Нет никакого сомнения, однако, что наши выводы в общих чертах сохранятся и для четырехмерной теории, которой мы надеемся посвятить отдельную статью.

Скажем здесь еще раз, что, как известно, последовательная формулировка релятивистской квантовой механики возможна только для свободного гамильтониана (по существу, в свое время это и послужило главной причиной создания квантовой теории поля). Тем не менее именно свободные частицы играют в квантовой теории ключевую роль, определяя асимптотические in- и out-состояния. Поэтому вопрос об альтернативных подходах к структуре асимптотических свободных полей не является, быть может, для квантовой теории поля праздным.

\section{2. ГРУППА ГАЛИЛЕЯ В ДВУМЕРНОМ ПРОСТРАНСТВЕ-ВРЕМЕНИ}

В начале этого раздела выразим галилееву функцию Вигнера $W_{\text {нер }}$ через волновую функцию $\widetilde{\psi}(\mathbf{q}, t)$ частицы в координатном представлении (4) и (5). С помощью преобразования Фурье, обратного к (4), выразим импульсные волновые функции через координатные и подставим соответствуюшие выражения в исходную формулу (3) для $W_{\text {нер. }}$ После этого внутренние интегралы по $d \mathbf{p}_{1}$ и $d \mathbf{p}_{2}$ легко вычисляются. В результате останется лишь интеграл по координатным переменным. После простых замен получим в итоге

$$
W_{\text {нер }}(\mathbf{p}, \mathbf{q} ; t)=\frac{1}{(2 \pi)^{3}} \int d \boldsymbol{\rho} \widetilde{\psi}^{*}\left(\mathbf{q}+\frac{\boldsymbol{\rho}}{2}, t\right) \widetilde{\psi}\left(\mathbf{q}-\frac{\boldsymbol{\rho}}{2}, t\right) e^{i \mathbf{p} \boldsymbol{\rho}} .
$$

В случае, когда пространство-время двумерно (т.е. его пространственная компонента одномерна), в формуле (11) изменится лиш численный множитель, а именно

$$
W_{\text {нер }}(p, q ; t)=\frac{1}{2 \pi} \int d \rho \widetilde{\psi}^{*}\left(q+\frac{\rho}{2}, t\right) \widetilde{\psi}\left(q-\frac{\rho}{2}, t\right) e^{i p \rho} .
$$

Формулу (11) мы будем называть координатным представлением функции Вигнера. Оно очень удобно для доказательства базовых свойств 1-4 нерелятивистской функции Вигнера. 
Передокажем сначала формулу (7). В соответствии с (11) находим

$$
\int d \mathbf{p} W_{\text {нер }}=\int d \boldsymbol{\rho} \delta(\boldsymbol{\rho}) \widetilde{\psi}^{*}\left(\mathbf{q}+\frac{\boldsymbol{\rho}}{2}, t\right) \widetilde{\psi}\left(\mathbf{q}-\frac{\boldsymbol{\rho}}{2}, t\right),
$$

откуда и вытекает соотношение (7).

Что касается равенства (6), то находим

$$
\begin{aligned}
\int d \mathbf{q} W_{\text {нер }}(\mathbf{p}, \mathbf{q} ; t)= & \frac{1}{(2 \pi)^{6}} \int d \mathbf{q} \int d \mathbf{p}_{1} d \mathbf{p}_{2} \psi^{*}\left(\mathbf{p}_{1}\right) \exp \left(-i \mathbf{p}_{1} \mathbf{q}+i \mathbf{p}_{2} \mathbf{q}\right) \psi\left(\mathbf{p}_{2}\right) \times \\
& \times \exp \left(i \frac{\mathbf{p}_{1}^{2}}{2 m} t-i \frac{\mathbf{p}_{2}^{2}}{2 m} t-i \mathbf{p}_{1} \frac{\boldsymbol{\rho}_{1}}{2}-i \mathbf{p}_{2} \frac{\boldsymbol{\rho}_{2}}{2}+i \mathbf{p} \boldsymbol{\rho}\right) d \boldsymbol{\rho}= \\
= & \int d \mathbf{p}_{1} d \mathbf{p}_{2} \delta\left(\mathbf{p}-\frac{\mathbf{p}_{1}+\mathbf{p}_{2}}{2}\right) \delta\left(\mathbf{p}_{1}-\mathbf{p}_{2}\right) \exp \left(-i \frac{\mathbf{p}_{1}^{2}-\mathbf{p}_{2}^{2}}{2} t\right)= \\
= & \psi^{*}(\mathbf{p}) \psi(\mathbf{p}) .
\end{aligned}
$$

Интегрирование по $d \mathbf{q}$ и $d \boldsymbol{\rho}$ дает две $\delta$-функции $\delta\left(\mathbf{p}_{1}-\mathbf{p}_{2}\right)$ и $\delta\left(\mathbf{p}-\left(\mathbf{p}_{1}+\mathbf{p}_{2}\right) / 2\right)$, которые и воспроизводят ожидаемьй ответ $\psi^{*}(\mathbf{p}) \psi(\mathbf{p})$ (эта величина не зависит от времени $t$ ).

Наконец, убедимся, что функция $W_{\text {нер }}(\mathbf{p}, \mathbf{q} ; t)$ зависит только от комбинации $\mathbf{q}-\mathbf{p} t / m$. В самом деле, согласно равенству (11) и выражению координатных волновых функций через импульсные получаем

$$
\begin{aligned}
W_{\text {нер }}(\mathbf{p}, \mathbf{q} ; t)= & \int d \mathbf{p}_{1} d \mathbf{p}_{2} d \boldsymbol{\rho} \frac{1}{(2 \pi)^{6}} \psi^{*}\left(\mathbf{p}_{1}\right) \psi\left(\mathbf{p}_{2}\right) \exp \left(i \mathbf{p}_{2} \mathbf{q}-i \mathbf{p}_{2} \frac{\boldsymbol{\rho}}{2}\right) \times \\
& \times \exp \left(-i \mathbf{p}_{1} \mathbf{q}-i \mathbf{p}_{1} \frac{\boldsymbol{\rho}}{2}\right) \exp (i \boldsymbol{\rho} \mathbf{p}) \exp \left\{i t \frac{\left(\mathbf{p}_{1}-\mathbf{p}_{2}\right)\left(\mathbf{p}_{2}+\mathbf{p}_{1}\right)}{2 m}\right\}= \\
= & \frac{1}{(2 \pi)^{3}} \int d \mathbf{p}_{1} d \mathbf{p}_{2} \delta\left(\mathbf{p}-\frac{\mathbf{p}_{1}+\mathbf{p}_{2}}{2}\right) \times \\
& \times \exp \left\{i \frac{\left(\mathbf{p}_{1}-\mathbf{p}_{2}\right)\left(\mathbf{p}_{1}+\mathbf{p}_{2}\right) t}{2 m}\right\} \exp \left\{i\left(\mathbf{p}_{2}-\mathbf{p}_{1}\right) \mathbf{q}\right\}= \\
= & W_{\text {нер }}\left(\mathbf{p}, \mathbf{q}-\frac{\mathbf{p}}{m} t\right)
\end{aligned}
$$

что мы и хотели показать.

Группа Галилея в двумерном пространстве включает следующие преобразования: а) сдвиги по времени на величину $\tau$, т.е. замены каждого события с временно́й и пространственной координатами $(t, q)$ событием с координатами $(t+\tau, q)$; б) пространственные трансляции на вектор $a$, т.е. замены каждого события с координатами $(t, q)$ событием с координатами $(t, q+a)$; в) переходы в подвижную систему отсчета, движушуюся в исходной с относительной скоростью $-v$, т.е. замены каждого события $(t, q)$ событием $(t, q+v t) ;$ г) комбинации перечисленных преобразований. Поскольку с точки зрения классического ансамбля вигнеровских псевдочастиц состояние физической системы полностью задается ее начальным положением, то в качестве представителя такового может быть выбрана сама функция Вигнера в любой фиксированный момент времени (стандартно мы будем считать, что для этого используется нулевое время $t=0$ ). 
При любых преобразованиях симметрии величина $W_{\text {нер }}(p, q ; 0)$ меняется по некоторому определенному закону

$$
W_{\text {нер }}(p, q ; 0) \rightarrow \widehat{W}_{\text {нер }}(p, q ; 0),
$$

в котором может быть, например, сохранена идея, что $W_{\text {нер }}$ - это псевдоплотность распределения псевдочастиц по фазовому пространству. Наша задача будет, таким образом, заключаться каждый раз в том, чтобы подобрать такое правило преобразования волновой функции, входящей в $W$, которое обеспечивало бы соотношение (15).

При временны́х трансляциях функция Вигнера меняется по правилу

$$
W_{\text {нер }}(p, q ; 0) \rightarrow W_{\text {нер }}^{\tau}(p, q ; 0)=W_{\text {нер }}(p, q ; \tau)
$$

а при пространственных - по правилу

$$
W_{\text {нер }}(p, q ; 0) \rightarrow W_{\text {нер }}^{a}(p, q ; 0)=W_{\text {нер }}(p, q+a ; 0) \text {. }
$$

Как мы уже отмечали, и время, и координата входят в функцию Вигнера только через упомянутый выше координатный фазовый множитель, включенный в функцию Вигнера. Поэтому понятно, что при временны́х трансляциях на величину $\tau$ закон преобразования волновой функции $\psi$ можно выбрать в виде

$$
\psi(p) \rightarrow \psi^{\tau}(p)=\psi(p) \exp \left(-i \frac{p^{2}}{2 m} \tau\right),
$$

а при пространственных трансляциях на вектор $a-$ в виде

$$
\psi(p) \rightarrow \psi^{a}(p)=\psi(p) \exp (i p a)
$$

Чтобы рассмотреть трансформационные свойства волновой функции по отношению к галилеевым бустам (т.е. к переходам к бесконечно медленным подвижным системам отсчета), введем преобразования Фурье фигурируюших здесь функций:

$$
\begin{aligned}
\widetilde{W}(\xi) & =\int d p W(p) e^{i p \xi} \\
\widetilde{\psi}(\xi) & =\int d p \psi(p) e^{i p \xi}
\end{aligned}
$$

В терминах этих фурье-преобразований и применительно к случаю двумерного пространства-времени наше базовое соотношение означает, что

$$
\widetilde{W}(2 \xi)=\frac{1}{2 \pi} \widetilde{\psi}^{*}(-\xi) \widetilde{\psi}(\xi)
$$

Это уравнение показывает, что при анализе его возможных решений сушественную роль должна играть следующая инволюция $\hat{I}$ комплексных функций переменной $\xi$ :

$$
\hat{I} \psi(\xi)=\psi^{*}(-\xi)
$$


Всякая комплексная функция $\psi(\xi)$ может быть однозначно разложена на симметричную (по отношению к этой инволюции) и антисимметричную части:

$$
\psi(\xi)=\frac{1}{2}\left[\psi(\xi)+\psi^{*}(-\xi)\right]+\frac{1}{2}\left[\psi(\xi)-\psi^{*}(-\xi)\right]
$$

Симметричную часть функции мы будем назьвать квазивешественной, а антисимметричную - квазимнимой. Эта терминология кажется естественной, поскольку каждую квазивешественную функцию можно рассматривать как преобразование Фурье некоторой вешественной функции, а каждую квазимнимую функцию - как преобразование Фурье чисто мнимой. Всякую комплексную функцию $\psi(\xi)$ можно представить в виде "полярного разложения"

$$
\psi(\xi)=\rho(\xi) e^{\phi(\xi)}
$$

где

$$
\rho(\xi)=\sqrt{\psi(\xi) \psi^{*}(-\xi)}
$$

- это (квазивещественньй) квазимодуль, а $\phi(\xi)$ - это (квазимнимая) квазифаза этой функции:

$$
\phi(\xi)=\operatorname{arcth} \frac{\psi(\xi)-\psi^{*}(-\xi)}{\psi(\xi)+\psi^{*}(-\xi)} .
$$

Если $\widetilde{\psi}(\xi)$ - это некоторое решение уравнения (18), то величина

$$
\widetilde{\psi}_{\phi}(\xi)=\widetilde{\psi}(\xi) e^{\phi(\xi)}
$$

тоже оказывается решением при любой квазифазе $\phi(\xi)$. Понятно, что этим и исчерпьвается произвол в определении $\widetilde{\psi}(\xi)$ через $\widetilde{W}(\xi)$. Чтобы подготовить соответствуюшие выкладки, касаюшиеся группы Лоренца, рассмотрим трансформационные свойства волновой функции по отношению к переходам в подвижную систему отсчета в инфинитезимальной форме.

При галилеевом бусте, заданном (бесконечно малой) относительной скоростью $-v$, функция Вигнера меняется по правилу

$$
W_{\text {нер }}(p, q ; 0) \rightarrow W_{\text {нер }}^{v}(p, q ; 0)=W_{\text {нер }}(p+m v, q ; 0)
$$

(в этом равенстве, разумеется, для нас существенны сейчас только члены порядка не

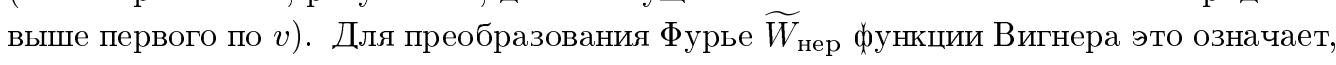
что

$$
\widetilde{W}_{\text {нер }}(\xi) \rightarrow \widetilde{W}_{\text {нер }}^{v}(\xi)=\widetilde{W}_{\text {нер }}(\xi) e^{-i m v \xi} .
$$

Уравнение (18) показывает, что соотношение (20) будет выполнено, если предположить, что при галилеевом бусте волновая функция $\widetilde{\psi}$ претерпевает изменение

$$
\widetilde{\psi}(\xi) \rightarrow \widetilde{\psi}^{v}(\xi)=\widetilde{\psi}(\xi)[1-i \xi m v]
$$

(опять же с точностью только до младших порядков по $v$ ). 
Действительно, с помощью (21) запишем аналогичное условие инвариантности еше и для величины $\widetilde{\psi}^{*}(-\xi)$ :

$$
\widetilde{\psi}^{*}(-\xi) \rightarrow \widetilde{\psi}^{v *}(-\xi)=\widetilde{\psi}^{*}(-\xi)[1-i \xi m v] .
$$

Теперь перемножим равенства (21) и (22), умножим результат на $1 / 2 \pi$ и удержим в нем только члены нулевого и первого порядков по $v$. Получим

$$
\widetilde{W}(2 \xi) \rightarrow \widetilde{W}^{v}(2 \xi)=\widetilde{W}(2 \xi)[1-2 i \xi m v] .
$$

В нужном приближении этот ответ согласуется с условием (21) галилеевой инвариантности функции Вигнера. Получая его, мы не использовали упомянутый ранее произвол, связанный с возможным инфинитезимальным изменением квазифазы в волновой функции при галилеевом бусте. Иными словами, мы предположили, что любая квазивешественная волновая функция остается при "бустировании" квазивешественной. Как видно, такой выбор является самосогласованным, а сам ответ совпадает с обшепринятым. Впрочем, для нас этот вывод полезен лишь тем, что позднее мы используем близкую технику для анализа трансформационных свойств волновой функции по отношению к группе Лоренца.

Чтобы восстановить глобальную формулу для преобразования волновой функции $\widetilde{\psi}(\xi)$ при переходе в подвижную систему отсчета, движущуюся с конечной скоростью $-v$, обозначим результат такого преобразования $\widetilde{\psi}(\xi \mid v)$. В силу условия $(21)$ величина $\widetilde{\psi}(\xi \mid v)$ удовлетворяет дифференшиальному уравнению

$$
\frac{d \widetilde{\psi}(\xi \mid v)}{d v}=-i \xi m \widetilde{\psi}(\xi \mid v) .
$$

Решение этого уравнения, отвечающее начальному условию

$$
\widetilde{\psi}(\xi \mid v)=\widetilde{\psi}(\xi)
$$

при $v=0$, задается формулой

$$
\widetilde{\psi}(\xi \mid v)=\widetilde{\psi}(\xi) \exp \{-i m \xi v\} .
$$

Возвращаясь с помощью обратного преобразования Фурье к исходной функции

$$
\psi(p \mid v)=\frac{1}{2 \pi} \int d \xi e^{-i p \xi} \widetilde{\psi}(\xi \mid v),
$$

получаем

$$
\psi(p) \rightarrow \psi(p \mid v)=\psi(p+m v) .
$$

Конечно же, этот ответ также был вполне предвидим. 


\section{3. ФУНКЦИЯ ВИГНЕРА $W(\mathbf{p}, \mathbf{q} ; t)$ В ДВУМЕРНОМ ПРОСТРАНСТВЕ-ВРЕМЕНИ И МАЛЫЕ ЛОРЕНЦЕВЫ ПОВОРОТЫ (БУСТЫ)}

В следуюшем разделе мы попытаемся извлечь информацию о законе преобразования релятивистской волновой функции исходя из априорных "естественных" правил преобразования функции Вигнера при лоренцевых "бустах". Сейчас же мы подробнее поговорим о том, какой закон преобразования $W(p, q, 0) \rightarrow \widehat{W}(p, q ; 0)$ мы считаем "естественным". Мы хотим здесь думать, что этот закон должен обеспечивать лоренц-инвариантность эволюции ансамбля вигнеровских частиц при его "бустировании" (т.е. замене его таким ансамблем, который с точки зрения исходной системы отсчета выглядит так же, как исходный ансамбль выглядит из системы отсчета, "повернутой” на соответствующий “обратный” бесконечно малый гиперболический угол). Пусть любое событие $(t, x)$, имеюшее в исходной системе отсчета временну́ю координату $t$ и пространственную координату $x$, при “бустировании” превращается в событие $(\hat{t}, \hat{x})$, где

$$
\hat{t}=t+q \nu, \quad \hat{x}=x+t \nu
$$

причем величина $\nu$ играет роль (релятивистской, хотя и бесконечно малой) скорости "бустирования".

УТВЕРЖДЕНИЕ. Для лорени-инвариантности динамической картинъ эволюиии ансамбля вигнеровских псевдочастии нужно, чтобь “бустированный” ансамбль описьвался функиией Вигнера $\widehat{W}(p, q ; 0)$ :

$$
W(p, q ; 0) \rightarrow \widehat{W}(p, q ; 0)=W(\hat{p}, \hat{q} ; 0),
$$

$2 \partial e$

$$
\hat{p}=p+\omega(p) \nu, \quad \hat{q}=q\left[1-\frac{p}{\omega(p)} \nu\right] .
$$

ДокАЗАТЕЛЬСтво. Для того чтобы понять, как преобразуется функция Вигнера, следует сначала разобраться, каким способом в результате "бустирования" изменяются в фазовой плоскости при $t=0$ координаты $p$ и $q$, задающие начальное положение траектории свободной псевдочастицы. Пусть в исходной системе отсчета некоторая траектория соединяет два события $\left(t_{1}, x_{1}\right)$ и $\left(t_{2}, x_{2}\right)$, причем очевидно, что

$$
x_{2}=x_{1}+\left(t_{2}-t_{1}\right) \frac{p}{\omega(p)}
$$

Ясно, что соответствуюшие “бустированные” события имеют в качестве двумерных координат следуюшие величины:

$$
\hat{t}_{1}=t_{1}+x_{1} \nu, \quad \hat{x}_{1}=x_{1}+t_{1} \nu
$$

и

$$
\hat{t}_{2}=t_{2}+x_{2} \nu, \quad \hat{x}_{2}=x_{2}+t_{2} \nu
$$


Отрезок траектории, соединяюший упомянутые события в исходной системе отсчета, задается параметрически соотношениями

$$
t=(1-\tau) t_{1}+\tau t_{2}, \quad x=(1-\tau) x_{1}+\tau x_{2},
$$

где параметр $\tau$ пробегает интервал $0<\tau<1$, а отрезок, соединяюший соответствуюшие "бустированные" события, - соотношениями

$$
\hat{t}=(1-\hat{\tau}) \hat{t}_{1}+\hat{\tau} \hat{t}_{2}, \quad \hat{x}=(1-\hat{\tau}) \hat{x}_{1}+\hat{\tau} \hat{x}_{2}
$$

где параметр $\hat{\tau}$ заключен в интервале $0<\hat{\tau}<1$. Нулевой момент времени $t=0$ согласно (31) отвечает значению параметра $\tau=\tau_{0}=-t_{1} /\left(t_{2}-t_{1}\right)$. При этом координата точки пересечения траектории с фазовой $(p, q)$-плоскостью при $t=0$ равна

$$
q=\frac{t_{2} x_{1}-t_{1} x_{2}}{t_{2}-t_{1}}
$$

(именно эта величина является одним из аргументов непреобразованной функции Вигнера). Что же касается "бустированной" (псевдо)частицы, то значение параметра $\hat{\tau}$, отвечающее времени, когда "бустированная" траектория попадает на интересную нам фазовую плоскость, задается соотношением

$$
\hat{\tau}=\hat{\tau}_{0}=\frac{t_{1}+q_{1} \nu}{\left(t_{1}-t_{2}\right)+\left(q_{1}-q_{2}\right) \nu} .
$$

В этот момент “бустированная" частица “пронизывает" фазовую плоскость в точке

$$
\hat{q}=q\left[1-\nu \frac{p}{\omega(p)}\right]
$$

что и является координатой, входящей в преобразованную функцию Вигнера. С другой стороны, понятно, что новая импульсная переменная возникает в соответствии с обычными преобразованиями Лоренца импульсов, т.е. равна

$$
\hat{p}=p+\omega(p) \nu
$$

Таким образом, под воздействием "бустирования" каждой вигнеровской псевдочастишы ее траектория, помеченная на фазовой плоскости при $t=0$ координатами $p$ и $q$, переходит в траекторию, “пронизываюшую" эту плоскость в точке с координатами $\hat{p}$ и $\hat{q}$. Естественно, плотность траекторий, т.е. функция Вигнера, подчиняется соответствующему индуцированному преобразованию. Тем самым утверждение доказано. Преобразования (29) другим способом были получены в работе [1] (где, однако, "бустирование" отвечало противоположному знаку относительной скорости двух систем отсчета). 


\section{4. ТРАНСФОРМАЦИОННЫЕ СВОЙСТВА ВОЛНОВЫХ ФУНКЦИЙ}

Обращаясь к релятивистской квантовой механике свободных частиц в двумерном пространстве-времени, рассмотрим симметрии, сохраняюшие лоренцев интервал между любыми парами событий. Как и в группе Галилея, такие симметрии включают, прежде всего, одномерные пространственные и временнь́е трансляции, и они могут быть изучены примерно такими же методами, что и группа Галилея (т.е. через классическую картину, связанную с функцией Вигнера). Возникающие при этом ответы весьма сходны с уже полученными в разделе 2 , а именно утверждается, что при временны́х трансляциях на величину $\tau$ волновая функция преобразуется по правилу

$$
\psi(p) \rightarrow \psi^{\tau}=\psi(p) e^{-i \omega(p) \tau}
$$

а при пространственных транслящиях на вектор $a-$ по правилу

$$
\psi(p) \rightarrow \psi^{a}=\psi(p) e^{i p a}
$$

Нетривиальный ответ возникает только при рассмотрении чисто лоренцева вращения, имеюшего неподвижную точку $t=0, q=0$ (все другие преобразования, сохраняющие интервал, могут рассматриваться как композиции таких вращений и пространственно-временнь́хх трансляций). Только этими нетривиальными лоренцевыми преобразованиями мы и будем в дальнейшем заниматься.

Опираясь на условия (26), (27) лоренц-инвариантности функции Вигнера $W(p, q ; t)$, найдем правила, которые обеспечивали бы эту инвариантность, для входящих в $W$ релятивистских волновых функций. Начнем с формул, связываюших в двумерном пространстве-времени величину $W(p, q ; t)$ с квантово-механическими волновыми функциями в импульсном представлении, а именно с функциями $\psi(p)$ на массовом гиперболоиде, отнесенными к моменту $t=0$. В работе [2] (см. там формулу (12)) было показано, что верно следуюшее соотношение:

$$
\begin{aligned}
W(p, q ; t)= & W(\operatorname{sh} \gamma, q ; t)=\frac{1}{\pi \operatorname{ch} \gamma} \int_{-\infty}^{+\infty} d \beta \operatorname{ch}(\gamma+\beta) \operatorname{ch}(\gamma-\beta) \times \\
& \times \psi^{*}(\operatorname{sh}(\gamma+\beta)) \psi(\operatorname{sh}(\gamma-\beta)) \exp \left\{-2 i\left[q-\frac{\operatorname{sh} \gamma}{\operatorname{ch} \gamma} t\right] \operatorname{sh} \beta \operatorname{ch} \gamma\right\}
\end{aligned}
$$

где положено $p \equiv \operatorname{sh} \gamma, \psi(p) \equiv \psi(\operatorname{sh} \gamma), m=1$. Мы воспользуемся этой формулой лишь затем, чтобы проиллюстрировать следуюшие два факта. Во-первых, из нее видно (на этот раз непосредственно), что переменные $q$ и $t$ входят в $W$ только через упомянутый во введении экспоненциальный координатный множитель $E$, причем переменные $q$ и $t$ появляются именно в комбинации $(q-p t / \omega(p))$. Во-вторых, нетрудно убедиться, что хотя в принципе этот координатный множитель может меняться как в результате изменения координаты $q$, так и в результате изменения импульса $p$, но при $t=0$ и при согласованном воздействии на $W$ двух малых преобразований $(27)$, связанных с “бустированием” 
при относительной скорости - $\nu$, эти два изменения взаимно компенсируются, так что в целом множитель

$$
\exp (-2 i q \operatorname{sh} \beta \operatorname{ch} \gamma)=\exp \{i q \operatorname{sh}(\gamma-\beta)-i q \operatorname{sh}(\gamma+\beta)\}
$$

остается постоянным. В самом деле, при преобразовании (27) этот координатный множитель принимает вид

$$
\begin{aligned}
E(\nu) & =\exp \{-2 i \hat{q} \operatorname{sh} \beta \operatorname{ch}(\gamma+\nu)\}= \\
& =\exp \left\{-2 i q\left(1-\nu \frac{\operatorname{sh} \gamma}{\operatorname{ch} \gamma}\right) \operatorname{sh} \beta \operatorname{ch} \gamma\left(1+\nu \frac{\operatorname{sh} \gamma}{\operatorname{ch} \gamma}\right)\right\} \simeq E(0)
\end{aligned}
$$

Таким образом, $\partial E / \partial \nu=0$, и для вычисления малой вариации функции $W(p, q ; 0)$ по отношению к преобразованиям (27) достаточно учитывать лишь вариацию импульсной переменной $\gamma$ всюду вне координатного множителя.

Другая рабочая формула, уже использованная в [1] (там она процитирована также под номером (12)), имеет вид

$$
\begin{aligned}
W(\operatorname{sh} \gamma, q ; 0)= & \frac{1}{2 \pi \operatorname{ch} \gamma} \int d \gamma_{1} d \gamma_{2} \operatorname{ch} \gamma_{1} \operatorname{ch} \gamma_{2} \delta\left(\gamma-\frac{\gamma_{1}+\gamma_{2}}{2}\right) \times \\
& \times \psi^{*}\left(\operatorname{sh} \gamma_{1}\right) \psi\left(\operatorname{sh} \gamma_{2}\right) \exp \left(i q \operatorname{sh} \gamma_{2}-i q \operatorname{sh} \gamma_{1}\right)
\end{aligned}
$$

(равенство (37) при $t=0$ и равенство (40) связаны заменой переменных $\gamma=\left(\gamma_{1}+\gamma_{2}\right) / 2$, $\left.\beta=\left(\gamma_{1}-\gamma_{2}\right) / 2\right)$.

Для выяснения вида малых преобразований волновой функции при "бустировании" заметим, что общая структура искомых формул нам уже вчерне известна - в работе [1] она была получена из условия лоренц-инвариантности функции Вигнера при $q=0$. Именно, в терминах фурье-преобразования

$$
\widetilde{\psi}(\xi)=\int e^{i \gamma \xi} \psi(\gamma) \operatorname{ch} \gamma d \gamma
$$

искомый инфинитезимальный закон должен иметь вид

$$
\widetilde{\psi}(\xi) \rightarrow \widetilde{\psi}^{\nu}(\xi)=\widetilde{\psi}(\xi)-\nu \widetilde{\Phi}(\xi) \widetilde{\psi}(\xi)
$$

где $\nu$ - это скорость бустирования, а функция $\widetilde{\Phi}(\xi)$ может иметь произвольную квазимнимую составляюшую $\tilde{A}(\underline{\xi})$, т.е. такую часть, что $\tilde{A}^{*}(-\xi)=-\tilde{A}(\xi)$, и фиксированную квазивешественную часть $\widetilde{R}(\xi)$, т.е. такую часть, что $\widetilde{R}^{*}(-\xi)=\widetilde{R}(\xi)$ :

$$
\widetilde{\Phi}(\xi)=\widetilde{R}(\xi)+\tilde{A}(\xi) .
$$

При этом

$$
\widetilde{R}(\xi)=\int d \eta \frac{1}{\widetilde{W}(2 \xi)} \Delta(\xi-\eta) \widetilde{W}(2 \eta)+i \xi
$$


Напомним, что здесь

$$
\Delta(\xi)=\int \frac{d \gamma}{2 \pi} \operatorname{th} \gamma e^{i \gamma \xi}
$$

Кроме того, $W(\gamma, q ; t)$ - это функция Вигнера в момент времени $t$ в точке фазового пространства $q, p=\operatorname{sh} \gamma$,

$$
\widetilde{W}(\xi)=\int d \gamma W(\gamma, 0 ; 0) \operatorname{ch} \gamma e^{i \gamma \xi}
$$

Напомним также, что при любой квазимнимой части $\tilde{A}(\xi)$ функции $\widetilde{\Phi}(\xi)$ и квазивешественной части $\widetilde{R}(\xi)$ этой функции, удовлетворяюшей соотношению $(44)$, вигнеровская псевдоплотность $W(\gamma, 0 ; 0)$ преобразуется в соответствии с правилом

$$
\widetilde{W}(\xi) \rightarrow \widetilde{W}^{\nu}(\xi)=\widetilde{W}(\xi)[1-i \nu \xi]-\nu \int d \eta \Delta(\xi-\eta) \widetilde{W}(\eta)
$$

В пересчете на исходную функцию $W(\gamma, 0 ; 0)$ закон (47) означает, что

$$
W(\gamma, 0 ; 0) \rightarrow W^{\nu}=W(\gamma+\nu, 0 ; 0)
$$

Таким образом, нам надлежит убедиться только в том, что квазимнимую функцию $\tilde{A}(\xi)$, фигурируюшую в (43), удастся выбрать таким образом, что функция Вигнера $W(\gamma, q ; 0)$ будет удовлетворять (ифинитезимально) полному условию лоренц-инвариантности, а именно условию

$$
W(\gamma, q ; 0) \rightarrow W^{\nu}(\gamma, q ; 0)=W(\gamma+\nu, q ; 0),
$$

где неизменность переменной $q$ на этот раз означает, что трансляции на величину $\nu$ подвергается только параметр $\gamma$ вне экспоненциального координатного множителя $E$. Последнее обстоятельство и является ключевым для проверки вьполнения общего условия лоренц-инвариантности функции Вигнера. Если при нулевой координате $q=0$ координатный множитель $E$ именно благодаря этому условию обрашался в единицу и исключался из конкурентной игры, могушей оказать влияние на правила преобразования волновой функции, то теперь появились другие причины, выраженные условием (39), для нейтрализации влияния фактора $E$. Поэтому неудивительно, что основные черты инфинитезимально преобразованной волновой функции удается получить из проведенного в работе [1] рассмотрения случая с нулевой координатой $q$. Так или иначе, мы утверждаем, что существуют такие (нелинейные) преобразования волновых функций, которые гарантируют выполнение полного условия лоренц-инвариантности функции Вигнера (49).

Тем не менее, по-видимому, полезно проследить подробно механизм реализации этого полного условия. Однако ввиду громоздкости соответствуюших выкладок и некоторой неявности возникающего при этом конкретного рецепта вычисления упомянутой выше квазимнимой функции $\tilde{A}(\xi)$ мы обсудим этот принципиально важный расчет в отдельной публикации. 
В заключение данного раздела обсудим возможный способ построения глобальных преобразований величин $\widetilde{\psi}(\xi)$. Допустим, что несмотря на неэксплицитность определения функции $\tilde{A}(\xi)$ мы все же сумели найти эту функцию. Тогда по ней можно в принципе построить глобальные преобразования, отвечающие инфинитезимальным правилам (42). Это можно сделать, например, так же, как мы поступили в рассмотренной выше задаче применительно к группе Галилея. Именно, обозначим через $\widetilde{\psi}\left(\xi \mid \nu_{0}\right)$ (глобально) преобразованную волновую функцию, отвечающую переходу к системе отсчета, которая движется относительно исходной со скоростью $-\nu_{0}$. Пользуясь инфинитезимальной формой, запишем следуюшую систему дифференциальных уравнений для функций $\widetilde{\psi}(\xi \mid \nu)$ :

$$
\frac{d \widetilde{\psi}(\xi \mid \nu)}{d \nu}=-\nu \widetilde{\psi}(\xi \mid \nu) \widetilde{\Phi}(\xi)
$$

с начальным условием

$$
\widetilde{\psi}(\xi \mid 0)=\widetilde{\psi}(\xi) .
$$

Проинтегрировав соответствуюшее решение от 0 до $\nu_{0}$, мы и должны получить (опять же в принципе) искомое глобальное преобразование

$$
\widetilde{\psi}(\xi) \rightarrow \widetilde{\psi}\left(\xi \mid \nu_{0}\right) .
$$

Благодарности. Я признателен Л. Д. Фаддееву за полезную критику. Данная работа выполнена при поддержке Российского фонда фундаментальных исследований (гранты № 99-01-00033, 99-01-0119D).

\section{Список литературы}

[1] О. И. Завьялов. Тр. МИАН. 2000. Т. 228. С. 136.

[2] О.И. Завьялов, А.М. Малокостов. ТМФ. 1999. Т. 119. С. 67.

[3] E. Wigner. Phys. Rev. 1932. V. 40. P. 749.

[4] T. Newton, E. Wigner. Rev. Mod. Phys. 1949. V. 21. P. 400.

[5] В. И. Татарский. УФН. 1983. Т. 139. С. 587. 